Ophthalmologica 1964;148:456-463

\title{
Index rerum ad Vol. 148
}

\section{Confecit F. Ammann, Geneve}

(B) = Buchbesprechungen - Livres nouveaux - Book reviews. $(\mathrm{R})=$ Referate von Vorträgen, die an anderer Stelle ausführlich erschienen sind oder erscheinen werden. Einzelheiten werden in diesem Index rerum nicht berücksichtigt. - Rapports de conferences qui ont été (ou seront) publiées ailleurs in extenso. Get Index rerum ne contient pas de details. - Abstract $\mathrm{s}$ of lectures that have been (or will be) published elsewhere. Details are not included in this Index rerum. $(\mathrm{V})=$ Vortrag - Communication - Report.

Abspaltungszonen, v. Diskontinui-

tätszonen, optische Akute Fälle; akute Fälle der täg-

lichen Praxis, für den dienst-

habenden Arzt, 454 (B) Allergie, v. Phlyktäne Alpha-Chymotrypsine, v. Star-operation

Amaurose, v. Ophthalmologische

Einführung, Schulung des Seh-

behinderten Amblyopie; ein neues Phänomen

Amblyoper mit exzentrischer

Fixation, 1 Amblyopie, v. Ophthalmologische

Einführung, Schulung des Seh-

behinderten Amotio retinae, v. Décollement

rétinien, Netzhautablösung, Retinal

detachment Anaesthesia; Fluothane anaesthesia

in glaucoma and cataract operations, 184 (V) Anesthesia; anesthesia in clinical

ophthalmology, 232 (B) Angiography; angiography in orbital

and peri-orbital pathology, 162 (V) Anterior chamber lens implants,

v. Vorderkammerlinsen Antigenic specificity of inclusion

bodies in epidemic keratoconjunc-

tivitis, 7 Aqueous, v. OrisuI Arteriitis temporalis, v. Ophthalmic

pathology

Augenbinnendruck, v. Anaesthesia, Anesthesia, Augeninnendruck, Glaucoma, Glaucomes, Iridenclei-sis, Photocoagulation

Augenheilkunde; Augenheilkunde. Leitfaden für Studium und Praxis (2. Aufl.), 312 (B)

-; Lehrbuch der Augenheilkunde, 231 (B)

Augenhintergrundsveränderungen, v. Chorioretinitis, serous central, Degeneratio maculae disciformis, Renal failure, Rétinopathíes dia-bétiques, Retinopathy

Augeninnendruck; Uveadurchblu-tung und Augeninnendruck, 312 (B)

Augenkomplikationen, v. Ghloro-quine, Cortisone, Cytomegalic inclusion disease, Nivaquine, Renal failure, Rougeole, Toxo-plasmose, Toxoplasmosis, Vorderkammerlinsen

Augenmuskeln, v. Diplopien, Muscles, Muskelsystem

Augenpuls, v. Oculo-sphygmography 
Barrière hémato-oculaire, v. Photo-coagulation Bindehaut, v. Conjunctiva Blepharospasmus; Beitrag zur neurochirurgischen Therapie des hartnäckigen Blepharospasmus, 130

Index rerum

ad Vol. 148

457

Blindheit, v. Ophthalmologische

Einführung, Rougeole, Schulung

des Sehbehinderten Blindness, v. Ophthalmologische

Einführung, Rougeole, Schulung

des Sehbehinderten Book reviews, 160 (B), 229-232 (B),

312 (B), 453-454 (B) Buchbesprechungen, 160 (B),

229-232 (B), 312 (B), 453-454 (B)

Calcium metastases, v. Sklera-mineralisation

Carcinoma of the kidney, v. Skleramineralisation

Carcinome renal, v. Skleramineralisation

Carotid artery; recording of arm-to-retina circulation-time by means of fundus reflectometry. A new photo-electric method for the diagnosis of internal carotid artery occlusion, 163 (V)

-; rubeosis of the iris associated with occlusion of the carotid artery, 196 (V)

Carotid artery, v. Oculo-sphygmo-graphy

Cat, v. Augeninnendruck

Cataract; Fluothane anaesthesia in glaucoma and cataract operations, 184 (V)

Cataract, v. Lens opacity, Star-operation

Cataracta traumatica, v. Lens opacity, Vorderkammerlinsen

Cécité, v. Ophthalmologische Einführung, Rougeole, Schulung des Sehbehinderten

Champ visuel, v. Nivaquine

Chat, v. Augeninnendruck

Chemotherapeutica et Antibiotica, v. Cortisone, Iodo-deoxyuridine, Orisul, Therapeutics, ocular,

Trihydroxyéthylrutoside

Chloroquine, v. Nivaquine

Chloroquine retinopathy, experimental, 442

Cholinesterase reactivators; stopping the action of strong miotics, 428

Chorioretinitis, serous central; the etiological agent of serous central chorioretinitis, 263

Ciliary hypotrichosis, congenital, 365

Complications oculaires, v. Chloroquine, Cortisone, Cytomegalic inclusion disease, Nivaquine,

Renal failure, Rougeole, Toxo-plasmose, Toxoplasmosis, Vorderkammerlinsen

Congrès, v. Ophthalmological Society

Congrès, annonce de, 160, 455

Conjunctiva; infectious diseases of the conjunctiva and cornea. Symposium of the New Orleans

Academy of Ophthalmology, 453 (B)

Cornea; infectious diseases of the conjunctiva and cornea. Symposium of the New Orleans

Academy of Ophthalmology, 453 (B)

Cornea, v. Iodo-deoxyuridine, Keratoconjunctivitis, epidemic, Rougeole

Cornea guttata; secondary cornea guttata in interstitial keratopathy, 289

Corneal preservation: A modified technique, 135

Cortisone; les formes cliniques des glaucomes dus à la Cortisone, 81 
Cortisone, v. Fasciculitis optica

Cristallin, v. Cataract, Diskonti-nuitätszonen, optische, Lens opacity, Vorderkammerlinsen

Cytomegalic inclusion disease; ophthalmic lesions due to cytomegalic inclusion disease, 98

Décollement rétinien; la pathogénie du décollement rétinien, 386

Décollement rétinien, v. Netzhaut-ablösung, Retinal detachment

Degeneratio maculae disciformis (Kuhnt-Junius), v. Ophthalmic pathology

Detachment of retina, v. Décollement rétinien, Netzhautablösung, Retinal detachment

Diagnosis, v. Carotid artery, Diplopien, Lymphangioma orbitae, Orbital pathology, Radio-logie, Radiology, Ultrasounds

Ophthalmologica, Vol. 148, No. 6 (1964)

;a

458

Index rerum

ad Vol. 148

Diplopien; einfache Methode zu $\gamma$ Analyse der Diplopien, 208 (V)

Diskontinuitätszonen, optische; ein Beitrag zuү Genese der optischen Diskontinuitätszonen der Augen-linse, 241

Doppelbilder, v. Diplopien

Echographie, v. Ultraschall, Ultrasounds

Einschlußkörperchen, v. Cytomegalic inclusion disease, Keratoconjuncti-vitis, epidemic

Electroretinogram, v. Nivaquine, Renal failure

Emergency, v. Akute Fälle

ERG, v. Electroret...

Errata, 232

Experimentelle Forschung, v. Chloroquine retinopathy, Chorioretinitis, serous central, Hypercholesteraemia, Orisul, Photocoagulation

Experiments in seeing, 312 (B)

Eye; malnutrition and the eye, 160 (B)

Eye injuries; injuries to the eye with lens opacity in young children, 169 (V)

Fascia lata in der Netzhautchirurgie. Vier Operationsmethoden, 271

Fasciculitis optica; Cortisone therapy in fasciculitis optica, 13

Fibrinolytic activity in ocular disease, 374

Fibrosarkom der Sklera, 416

Fixation, exzentrische, v. Amblyopie

Fluothane, v. Anaesthesia

Fortbildung und Orientierung, 219, 386

Fossette du nerf optique, v. Retinal detachment

Fukala-Operation; zur Voraussage der Refraktion und des Ver-größerungseffektes nach Fukala-

Operation auf Grund von Ultra-schalluntersuchungen, 393

Fundus, v. Chorioretinitis, serous central, Degeneratio maculae disciformis, Renal failure,

Rétino-pathies diabétiques, Retinopathy

Fundus reflectometry; recording of arm-to-retina circulation-time by means of fundus reflectometry. A new photo-electric method for the diagnosis of internal carotid artery occlusion, 163 (V) 
Further studies and other information, 219, 386

Gefäße, v. Arteriitis temporalis, Carotid artery, Orbital pathology, Renal failure

Gesichtsfeld, v. Nivaquine

Glaucoma; Fluothane anaesthesia in glaucoma and cataract operations, 184 (V)

Glaucoma, v. Iridencleisis, Lamina cribrosa

Glaucomes; les formes cliniques des glaucomes dus à la Cortisone, 81

Goniotomy; demonstration, by means of television, of a goniotomy using a newly-designed contact lens, $187(\mathrm{~V})$

Graft, corneal; our youngest case of a successful corneal graft, 425

Graft, corneal, v. Corneal preservation

Greffe cornéenne, v. Corneal preservation, Graft, corneal

Grubenpapille, v. Retinal detachment

Haute myopie, v. Ultraschall

Heredität, v. Ciliary hypotrichosis, congenital

Herpetic keratitis, v. Iodo-deoxyuridine

High myopia, v. Ultraschall

Histologie, v. Arteriitis temporalis, Chloroquine retinopathy, Chorioretinitis, serous central,

Cornea guttata, Degeneratio maculae disciformis, Fibrosarkom der Sklera, Lamina cribrosa,

Lym-phoid tumour, Skleraminerali-sation

Hornhaut, v. Cornea, Cornea guttata, Iododeoxyuridine, Keratoconjunc-tivitis, epidemic,

Rougeole

Hornhautplastik, v. Corneal preservation, Graft, corneal

Humor aqueus, v. Orisul

Index $\Gamma$ erum

ad Vol. 148

459

Hypercholesteraemia; the ocular manifestations of experimental hypercholesteraemia in rabbits, 57

Hypertension oculaire, v. Anaesthesia, Anesthesia, Glaucoma, Glau-comes, Iridencleisis Hypotension oculaire; Thypotension oculaire consecutive à la photo-coagulation du spincter de Гiris, 296

Inclusion bodies, v. Cytomegalic inclusion disease, Keratoconjunc-tivitis, epidemic

Inclusions cytomégaliques, v. Cytomegalic inclusion disease

Inclusions cytoplasmiques, v. Kera-toconjunctivitis, epidemic

Infectious diseases of the conjunctiva and cornea. Symposium of the New Orleans Academy of Ophthalmology, 453 (B)

Infektionslehre, klinische (3. Aufl.), 229 (B)

Inferior oblique muscle; antero-position of the inferior oblique muscle in V-esotropia, 325

Instrument, v. Goniotomy, Oculo-sphygmography

Intraocular pressure, v. Anaesthesia, Anesthesia, Augeninnendruck, Glaucoma, Glaucomes, Iridencleisis, Photocoagulation

Intraokularer Druck, v. Anaesthesia, Anesthesia, Augeninnendruck, Glaucoma, Glaucomes, Iridencleisis, Photocoagulation

Iodo-deoxyuridine; tolerance et activité de la 5-Iodo-2'-deoxy-uridine en ophtalmologie, 25 Iridencleisis; on iridencleisis, 213 (V) 
Junius-Kuhnt, v. Degeneratio maculae disciformis

Kalkmetastasen, v. Skleraminerali-

sation Kammerwasser, v. Orisul Kaninchen, v. Augeninnendruck, Chorioretinitis, serous central, Hypercholesteraemia, Photocoagulation

Katarakt, v. Lens opacity, Star-operation

Katze, v. Augeninnendruck

Kératite, v. Rougeole

Kératite herpétique, v. Iodo-deoxyuridine

Kératoconjonctivite phlycténulaire, v. Meteorotropie

Keratoconjunctivitis, epidemic; antigenic specificity of inclusion bodies in epidemic

keratoconjunctivitis, 7

Keratopathie, v. Vorderkammer-linsen

Keratopathy, interstitial, v. Cornea guttata

Keratoplasty, v. Corneal preservation, Graft, corneal

Kitahara, v. Chorioretinitis, serous central

Kongresse, v. Ophthalmological Society

Kongreßkalender, 160, 455

Kuhnt-Junius, v. Degeneratio maculae disciformis

Lamina cribrosa; histologische Untersuchung der Lamina cribrosa bei Glaukom, 431

Lapin, v. Augeninnendruck, Chorioretinitis, serous central, Hypercholesteraemia,

Photocoagulation

Lederhaut, v. Sklera

Lens, v. Cataract, Diskontinuitäts-zonen, optische, Vorderkammer-linsen

Lens opacity; injuries to the eye with lens opacity in young children, 169 (V)

Lentilles camérulaires, v. Vorder-kammerlinsen

Leptospirenuveitis, 91

Libri, 160 (B), 229-232 (B), 312 (B), 453-454 (B)

Lichtkoagulation, v. Light coagulation, Photocoagulation

Light coagulation; Symposium on research in light coagulation held in Richmond, Virginia,

March 1963 (Acta Ophthalmologica Suppl. 76), 454 (B)

Light coagulation, v. Photocoagulation

460

Index rerum

ad Vol. 148

Linse, v. Cataract, Diskontinuitäts-

zonen, optische, Vorderkammer-

linsen Livres nouveaux, 160 (B),

229-232 (B), 312 (B), 453-454 (B) Lymphangioma orbitae, ein Fall, 405 Lymphoid tumour of

the orbit,

v. Ophthalmic pathology

Maculaloch, v. Netzhautablösung, Betinal detachment

Macular hole, v. Netzhautablösung, Retinal detachment

Malnutrition and the eye, 160 (B)

Masern, v. Rougeole

Masuda-Kitahara, v. Chorio-retinitis, serous central 
Measles, v. Rougeole

Medical meetings, announcement of, 160, 455

Métastases calcaires, v. Sklera-mineralisation

Meteorotropie; Untersuchungen zur Frage der Meteorotropie von Phlyktänen, 233

Methode, v. Angiography, Corneal preservation, Fascia lata, Fundus reflectometry,

Netzhautablösung, Oculo-sphygmography, Slit lamp photography, Ultraschall, Ultrasounds

Miotics; stopping the action of strong miotics, 428

Morbilli, v. Rougeole

Muscles; surgery of the vertical muscles of the eye (2nd ed.), 230 (B)

Musculus obliquus inferior, v. Inferior oblique muscle

Muskelsystem; die Rolle des Muskel-systems in der Pathogenese und Therapie des Schielens, 230 (B)

Myopie, hochgradige, v. Ultraschall

Necrologia; Professor Dr. K. Safar

zum Gedenken, 80 Nephropathy, v. Retinal signs Nervus opticus, v. Fasciculitis optica

Netherlands Ophthalmological

Society, 151st Meeting (1963),

162-215 (V) Netzhautablösung; eine Operations-

methode für maculalochbedingte

Netzhautablösungen, 42

Netzhautablösung, v. Décollemenl rctinien, Netzhautchirurgie, Retinal detachment

Netzhautchirurgie; Fascia lata in der Netzhautchirurgie. Vier Opera-tionsmethoden, 271 Neuroophtalmologie; precis d'ophtalmologie et de neuro-ophtalmologie (3e éd.), 229 (B)

Nierenaffektionen, v. Retinal signs Nierenkrebs, v. Skleramineralisation Nivaquine; retinopathy resulting from prolonged use of Nivaquine, $188(\mathrm{~V})$

Occlusion of carotid artery,

v. Fundus reflectometry, Oculo-sphygmography, Rubeosis of the iris

Ocular pulse, v. Oculo-sphygmography

Ocular tension, v. Anaesthesia, Anesthesia, Augeninnendruck, Glaucoma, Glaucomes,

Iridenclei-sis, Photocoagulation

Oculo-sphygmography; a handy instrument for oculo-sphygmography with some clinical results, 367

Operation, v. Blepharospasmus, Cataract, Eye injuries, Gonio-tomy, Inferior oblique muscle, Iridencleisis, Netzhautablösung, Netzhautchirurgie, Staroperation, Vertical muscles,

Vorderkammer-linsen

Ophtalmologie; elements d'ophtalmologie, 160 (B)

-; precis d'ophtalmologie et de neuro-ophtalmologie (3e éd.), 229 (B)

Ophthalmic pathology; some aspects of ophthalmic pathology, 201 (V)

Ophthalmological Society, American, transactions (98th Meeting, 1962), 229 (B)

Ophthalmological Society, Netherlands, 151st Meeting (1963), 162-215 (V)

Ophthalmological Society of the United Kingdom, 84th congress (1964), 216-218 (R)

Ophthalmologie, v. Augenheilkunde

Index rerum

ad Vol. 148

461 
Ophthalmologische Einführung, 221 Optic neuritis, v. Fasciculitis optica Orbita; ein Fall von Lymphangioma

orbitae, 405 Orbita, v. Lymphoid tumour of the

orbit Orbital pathology; angiography in

orbital and peri-orbital pathology,

162 (V) Orisul; experimentelle Untersuchun-

gen über das Diffusionsvermögen

von Orisul ins Vorderkammer-

wasser von Kaninchen, 302 Oximes, v. Cholinesterase

reactivators

PAM, v. Cholinesterase reactivators

Papillitis, v. Fasciculitis optica

Pathogenese, v. Schielen

Perfectionnement et orientation, 219, 386

Pharmacology, ocular; handbook of ocular therapeutics and pharmacology, 453 (B)

«Phénomène du retournement», v. Amblyopie

Phlyktäne; Untersuchungen zur Frøge der Meteorotropie von Phlyktänen, 233

Photocoagulation; Thypotension oculaire consecutive à la photo-coagulation du sphincter de

Гiris, 296

Photocoagulation, v. Light coagulation

Photographie à la lampe à fente, v. Slit lamp photography

Physiology, v. Experiments in seeing

Pit of optic disc, v. Retinal detachment

Plasmin activity, v. Fibrinolytic activity

Pouls oculaire, v. Oculo-sphygmo-graphy

Pression oculaire, v. Anaesthesia, Anesthesia, Augeninnendruck, Glaucoma, Glaucomes, Iridencleisis, Photocoagulation

Prize; Heinz Karger Memorial Prize, 455

-; International Eye Film Festival, Chicago (October, 1965), 455

Rabbit, v. Augeninnendruck, Chorio-retinitis, serous central, Hyper-cholesteraemia, Photocoagulation

Radiologie; Handbuch der medizini-schen Radiologie, Vol. VII/2: Rönt-gendiagnostik des

Schädels II, 453 (B)

Radiology; encyclopedia of medical radiology, vol. VII/2: Roentgen diagnosis of the skull II, 453 (B)

Radioterapia delle affezioni oculari, 231 (B)

Rat, v. Chloroquine retinopathy

Ratte, v. Chloroquine retinopathy

Recherches expérimentales, v. Chloroquine retinopathy, Chorioretinitis, serous central,

Hypercholesterae-mia, Orisul, Photocoagulation

Refraktion, v. Fukala-Operation

Renal failure; new retinal signs of renal failure, 342

Retinal detachment; macular and juxtapapillar serous retinal detachment associated with pit of optic disc, 247

Retinal detachment, v. Décollement rétinien, Netzhautablösung

Retinal signs; new retinal signs of renal failure, 342 
Rétinopathies diabétiques: Possi-bilités et limites du traitement par des doses élevées d'un facteur $\mathrm{P}$, le Trihydroxyéthylrutoside, 121

Retinopathy; experimental Chloroquine retinopathy, 442

-; retinopathy resulting from prolonged use of Nivaquine, $188(\mathrm{~V})$

Retrobulbar neuritis, v. Fasciculitis optica

Rougeole; les complications oculaires de la rougeole, cause majeure de cécité chez 1'enfant en pays tropical, 107

Rubeosis of the iris associated with occlusion of the carotid artery, 196 (V)

Safar, K.; Professor Dr. K. Safar zum Gedenken, 80

Schielen; die Rolle des Muskel-systems in der Pathogenese und Therapie des Schielens, 230 (B)

462

Index rerun!

ad Vol. 148

Schielen, v. Diplopien, Inferior oblique muscle, Muscles, Stra-bisme, Strabismus

Schulung des Sehbehinderten, 225

Sclérotique, v. Sklera

Sehschwäche, v. Ophthalmologische Einführung, Schulung des Sehbehinderten

Sitzungsberichte, 216-218 (R)

Sklera; Fibrosarkom der Sklera, 416

Skleramineralisation; Kalkmetasta-sen der Sklera bei primärem Nie-renkrebs. Ein Beitrag zur

Pro-blematik der Skleramineralisation, 252

Slit lamp photography; depth of field in slit lamp photography: An optical solution using the

Scheimpflug principle, 143

Socités, comptes rendus, 216-218 (R)

Societies, v. Ophthalmological Society

Society transactions, 216-218 (R)

Spaltlampenphotographie, v. Slit lamp photography

Sphincter de $\Gamma$ iris, v. Photocoagu-lation

Squint, v. Diplopien, Inferior oblique muscle, Muscles, Schielen, Stra-bisme, Strabismus

Star, grauer, v. Cataract, Lens opacity, Staroperation

Staroperation; die Staroperation mit fermentativer Lösung der Zonula, 230 (B)

Statistische Auswertung, v. Fasciculitis optica, Inferior oblique muscle, Nivaquine, Rétinopathies

diabétiques, Strabisme, Vorder-kammerlinsen

Strabisme; résultats fonctionnels actuels dans le traitement du strabisme - statistiques 1963, 313

Strabisme, v. Diplopien, Inferior oblique muscle, Muscles, Schielen, Strabismus

Strabismus. Symposium of the New Orleans Academy of Ophthalmology, 231 (B)

Sulfonamid, v. Orisul

Tension oculaire, v. Pression oculaire

Therapie, v. Eye injuries, Fasciculitis optica, Glaucoma, Iodo-deoxyuri-dine, Leptospirenuveitis, Lymph-angioma orbitae, Radioterapia, Rétinopathies diabétiques, Schielen, Strabisme

Therapeutics, ocular; handbook of ocular therapeutics and pharmacology, 453 (B)

Toxoplasmose; la toxoplasmose et ses manifestations oculaires, 232 (B)

Toxoplasmosis, argumenta et docu-menta ophthalmologica, 231 (B)

Transactions of the American Ophthalmological Society (98th Meeting, 1962), 229 (B)

Trauma, v. Lens opacity 
Trihydroxyéthylrutoside, v. Rétinopathies diabétiques

Trou maculaire, v. Netzhautablö-sung, Retinal detachment

Tumor, v. Fibrosarkom der Sklera, Lymphangioma orbitae, Lymphoid tumour of the orbit

Ultraschall; zur Voraussage der Refraktion und des Vergröße-rungseffektes nach Fukala-

Operation auf Grund von Ultra-schalluntersuchungen, 393

Ultraschall, v. Ultrasounds

Ultrasons, v. Ultraschall, Ultrasounds

Ultrasounds; the possibilities of ultrasonic localization as a diagnostic aid in ophthalmology, 151

«Umkehrphänomen», v. Amblyopie

United Kingdom, v. Ophthalmological Society

Untersuchungstechnik, v. Angio-graphy, Fundus reflectometry, Oculo-sphygmography, Radiologie, Ultraschall, Ultrasounds

Urgence, v. Akute Fälle

Uveadurchblutung und Augeninnen-druck, 312 (B)

Uveitis; über Leptospirenuveitis, 91

Vaisseaux, v. Arteriitis temporalis, Carotid artery, Orbital pathology, Renal failure

Varia, 455

Index rerunt ad Vol. 148

463

VenoГuton P4, v. Rétinopathies

diabétiques Vererbung, v. Ciliary hypotrichosis, congenital Vergrößerungseffekt, v. Fukala-

Operation Vertical muscles; surgery of the

vertical muscles of the eye (2nd

ed.), 230 (B) Vessels, v. Arteriitis temporalis,

Carotid artery, Orbital pathology,

Renal failure Virus, v. Chorioretinitis, serous

central, Cytomegalic inclusion

disease, lodo-deoxyuridine,

Keratoconjunctivitis, epidemic Vision, v. Experiments in seeing

Vision, binocular; contribution to

the study of disturbed binocular

vision, 187 (V) Visual field, v. Nivaquine Vorderkammerlinsen; Ergebnisse

nach Vorderkammerlinsenimplan-

tation, 355

Xanthomatosis, ocular, v. Hyper-cholesteraemia

Zones de discontinuité optique, v. Diskontinuitätszonen, optische

Zones of optical discontinuity, v. Diskontinuitätszonen, optische

Zonulolyse, fermentative, v. Staroperation

Index autorum ad Vol. 148

Confecit F. Ammann, Geneve

(B) = Buchbesprechungen - Livres nouveaux - Book reviews $(\mathrm{R})=$ Referate von Vorträgen, die an anderer Stelle ausführlich erschienen sind oder erscheinen werden. Die Autorennamen wurden in diesem Index autorum nicht aufgenommen. - Rapports de conferences qui ont été (ou seront) publiées ailleurs in extenso. Cet Index autorum ne contient pas les noms des auteurs. - 
Abstracts of lectures that have been (or will be) published in extenso elsewhere. The names of the authors have not been included in this Index autorum. $(\mathrm{V})=$ Vortrag - Communication Report.

Adlakha, D. D, 374 Aichmair, H., 1 Asher, H., 312 (B)

Balestra, E., 231 (B) Beintema, D. K., 163 (V) Binkhorst, C. D., 169 (V) Bobiński, H., 302

Boen-Lian, Sie, v. Sie-Boen-Lian Boles-Carenini, B., 231 (B) Bouzas, A., 25 Brandt, H. P., 233

Bynke, H. G., 367

Charan, H., 365 Cuendet, J. F., 121 Czerek-Jaguczańska, H., 151

Dán, P., 7

Dekking, H. M., 428 Deller, M., 230-231 (B) Delorme, A., 229 (B) Demaret, M., 296 De Raad, R., 162 (V) Diethelm (Editor), 453 (B) Donders, P. C, 201 (V) Drews, R. C, 143 Dufour, R., 386 Duncalf, D., 232 (B) Dvorak, M., 130

E. Ellis, P. P., 453 (B)

Fabiszewska-Górny, D., 241 Fink, W. H., 230 (B) 